\title{
Assumption of heparin-induced thrombocytopenia in mucopolysaccharidoses
}

\section{Liliya Aleksandrovna Osipova ${ }^{* *}$, Lyudmila Mikhailovna Kuzenkova²}

\begin{abstract}
Background: Mucopolysaccharidoses (MPSs) are a group of lysosomal storage diseases, resulted from glycosaminoglycans' breakdown failure (GAGs). The study aims to determine the presence of thrombocytopenia, its prevalence, clinical implication, and correlation with the types of MPSs and with the types of glycosaminoglycans storage.
\end{abstract}

Methods: A retrospective cross-sectional study of complete blood count data was conducted among 108 children with a confirmed diagnosis of MPS in the National Medical Research Center for Children's Health, Moscow, Russian Federation. STATISTICA 10 (Stat Soft, Inc. 1984-2011) was used for statistical analysis. A p-value of $\leq 0.05$ was considered significant.

Results: The median age of children was 65 [IQR: 41; 102] months, range: $3-102$ months. The male to female ratio was 3:1. The prevalence of fluctuating or persistent thrombocytopenia in all children with MPS was $19.0 \%$.

Thrombocytopenia occurred in patients with MPS I (14.0\%), II (19.0\%), and III (31.0\%). Neither of the patients with MPS IV nor VI demonstrated low platelet count. There was a non-significant predominance $(P=0.068)$ of thrombocytopenia frequency in patients with heparan sulfate storage $(22.0 \%)$ compared with patients without heparan sulfate burden (0\%).

Conclusion: Routine checkups of patients with MPSs must include complete blood count with platelet measurement. Cases of thrombocytopenia that requiring treatment, immunomodulatory/ immunosuppressive therapy should be considered. Further research is needed to look for the laboratory confirmation of autoimmune variants of HIT in patients with MPS I, II, III.

Keywords: Mucopolysaccharidoses, Glycosaminoglycans' Storage, Heparan Sulfate, Autoimmune Thrombocytopenia, Moscow, Russian Federation

\section{Background}

Mucopolysaccharidoses (MPSs) are a group of multisystem metabolic diseases resulting from deficiency of the Lysosomes enzymes involved in glycosaminoglycans' breakdown. The classical clinical picture of mucopolysaccharidoses does not comprise thrombocytopenia. Nevertheless, less increasing number of case reports describing thrombocytopenia in patients with Hunter and Sanfilippo syndromes [1-9] and even in a child with unspecified type of MPS [10] have appeared in recent years. Some of the articles described clinical manifestations of thrombocytopenia, including serious complications: intracranial hemorrhage, recurrent epistaxis, melena and anemia, extradural hemorrhage following ventriculoperitoneal shunt insertion, intraoperative bleeding during adenoidectomy, epistaxis, and

*Correspondence: liliya-o@yandex.ru

${ }^{1}$ Consultative-diagnostic center, Federal State Autonomous Institution of the Ministry of Health of the Russian Federation "National Medical Research Center for Children's Health", Moscow, Russian Federation.

Full list of author information is available at the end of the article skin hemorrhages $[1,3,6,7,8,10]$. Notably, most cases of low platelet count were encountered in patients with heparan sulfate breakdown failure (namely MPS II and III). Thus, the present study aims to analyze thrombocytopenia prevalence, its clinical implication, and correlation with the MPSs' and glycosaminoglycans' types in a rather large group of pediatric patients.

\section{Methods}

\section{Design of study}

A retrospective cross-sectional study was conducted from January 2007 to December 2016 at the National Medical Research Center for Children's Health to reveal thrombocytopenia in a group of children with MPSs $(n=108)$.

Inclusion and exclusion criteria

All children with enzymatically and molecularly confirmed diagnoses of one of the MPSs' types were included in the study. 
There were no exclusion criteria for the patients with confirmed MPS.

\section{Procedures}

Data on complete blood count were retrospectively searched through the medical charts, recorded in National Medical Research Center for Children's Health, and through medical records provided from regional medical institutions. Clinical implications of thrombocytopenia were thoroughly explored through all medical charts. One or more complete blood count measures were available for each of the 108 children. All samples independently of the presence of pathogenic therapy (regular enzyme-replacement therapy or exposure to hematopoietic stem cell transplantation) were used for analysis. However, we excluded the measures obtained during acute respiratory infections, after epileptic status or surgery, and those low platelet count measures suspicious of adverse drug events.

\section{Definition of variables}

Patients having one or more platelets count less than 150x109/L were considered thrombocytopenia cases. To evaluate the influence of the type of accumulated glycosaminoglycan on the development of thrombocytopenia, we analyzed the prevalence of thrombocytopenia in subgroups of patients with different types of MPSs. In respect to all published cases of thrombocytopenia included only patients with MPS II and III, we separately compared the prevalence of discussed disturbance in the subgroups of patients with and without heparan sulfate storage that is considered to have structural and functional similarity to heparin.

\section{Statistical analysis}

We analyzed the frequency and values of thrombocytopenia in the whole group of children, in patients with different types of MPSs, and the groups with and without heparan sulfate storage. For descriptive statistic calculations, we used the minimal values of platelets' count (fixed for every patient during dynamic observation). Prevalence of thrombocytopenia in different types of MPSs was represented as absolute and relative frequency. Descriptive statistics of platelet count were expressed as medians, interquartile ranges, minimum and maximum. Fisher's two-tailed exact test was used to evaluate the difference between thrombocytopenia frequency in different types and groups of MPSs. We used STATISTICA 10 (Stat Soft, Inc. 1984-2011) for calculations. A P-value of $\leq 0,05$ was considered statistically significant.

\section{Results}

One hundred eight children with enzymatically and molecularly confirmed diagnosis of one of the MPSs' types were evaluated. 9 patients had MPS I Hurler, 4 - MPS I Hurler-Scheie, 1 -MPS I Scheie, 53 - MPS II, 26 - MPS III, 6 - MPS IVA, 9 - MPS VI. There were 26 female and 82 male patients. All patients with MPS II were males. The median age of children at the time of the first admission to the National Medical Research Center for Children's Health was 65 [IQR: 41; 102] months, minimum -3 months, maximum -102 months. Out of 108 children 20 $(19.0 \%)$ demonstrated fluctuating or persistent thrombocytopenia. There was no significant difference in the frequency of thrombocytopenia among different types of MPSs (Table1).

Table 1 Thrombocytopenia in children with different types of mucopolysaccharidoses $(\mathrm{N}=108)$

\begin{tabular}{|l|l|l|l|}
\hline Type of MPS & Number of patients & Frequency of thrombocytopenia ${ }^{\S}$ & ${\text { Platelet count, } x 10^{9} / \mathrm{L}^{*}}^{*}$ \\
\hline MPS I & 14 & $2 / 14(14 \%)$ & $77 ; 0$ \\
\hline MPS II & 53 & $10 / 53(19 \%)$ & $\begin{array}{l}135[116 ; 139] \\
98-145\end{array}$ \\
\hline MPS III & 26 & $8 / 26(31 \%)$ & $\begin{array}{l}107[89 ; 134] \\
82-147\end{array}$ \\
\hline MPS IVA & 6 & $0 / 6(0 \%)$ & - \\
\hline MPS VI & 9 & $0 / 9(0 \%)$ & - \\
\hline
\end{tabular}

Footnote.*median $[\mathrm{IQR}]$, min-max platelet count, that is beyond the reference range. §Comparing frequency in different types does not show significant difference.

Although apparent excess of thrombocytopenia frequency, however there was no statistically significant difference $(\mathrm{p}=0.068)$ among group of patients with MPSs, in which heparan sulfate breakdown failure occurs $(22.0 \%)$, compared with those without heparan sulfate storage $(0 \%)$ (Table 2). Only 4 of $20(20.0 \%)$ children, who had thrombocytopenia, experienced compatible clinical signs, presented as bleeding after tooth extraction in a girl with MPS IIIA, easy bruising and subdural hemorrhage - in a male with MPS IIIB, skin hemorrhages, and hematochezia in the neonatal period - in a male with MPS II. A male with Hurler syndrome, 2.5 months old, had a platelet count drop to an undetectable level; the clinical signs consisted of petechial rash. Clinical and laboratory manifestations resolved after intravenous immunoglobulin therapy.

\section{Discussion}

Despite a growing number of case reports describing thrombocytopenia in patients with MPSs, there is no single opinion about its pathogenic mechanism. Hypersplenism and substrate storage in bone marrow, as in Gaucher disease, are supposed to be the main causal factors by some researchers [4, 11-13]. To the best of your knowledge Us B. et al. [9] were the first and the only to consider an immune-mediated mechanism and/or possibly chronic antigenic stimulation due to GAG accumulation as the etiological factor of thrombocytopenia in MPS II. For the purpose of evolving our conception and summarizing the facts, a further discussion would be in this section considering the heparin-induced etiology of thrombocytopenia in MPSs. Heparin-induced thrombocytopenia (HIT) has not yet been discussed and explored in patients with these metabolic diseases. HIT is an immune prothrombotic 
complication of heparin therapy caused by antibodies to complexes of platelet factor 4 (PF4) and heparin. HIT antibodies may recognize PF4 bound to platelet chondroitin sulfate. Activation of platelets even in the absence of heparin, can explain spontaneous HIT syndrome (which resembles HIT clinically and serologically but occurs without proximate heparin exposure) [14]. Explanation of HIT possibility in MPSs can be structured as follows. Since the published case reports and our data have demonstrated the occurrence of thrombocytopenia exclusively in patients with heparan sulfate storage (MPS I, II, and III), heparan sulfate may play a significant role. As heparan sulfate is known to have structural and functional similarity to heparin [15-17], a well-known entity, called HIT, should be considered. Most research $[1,6,7,9,10]$ reported the efficacy of immunomodulatory/immunosuppressive therapy (intravenous immunoglobulin and corticosteroids) for thrombocytopenia in MPS II and III patients and encountered in one of our patients, is in accordance with HIT. Furthermore, cases of spontaneous HIT after orthopedic surgery have been described and judged to be the result of glycosaminoglycans' release, triggering the generation of complexes with PF4 and subsequent antibody production [18-22]. In addition, the emergence of thrombocytopenia after cessation of enzyme replacement therapy in two patients with Hunter syndrome, presented by Jurecka A, et al. [23] point out to the link between hematologic disturbance and increased glycosaminoglycans' amount in the blood.

Thus, the autoimmune variant of HIT is a probable manifestation of MPSs with heparan sulfate storage. This theoretical suggestion determines the need to investigate antibodies to PF4/heparin complexes, heparin-induced platelet aggregation, and serotonin release in patients with MPS I, II, and III. Determination of other predisposing factors, such as HLA alleles and killer-cell immunoglobulin-like receptors [23], would be of great interest.

Table 2 Heparan sulfate influence on the development of thrombocytopenia $(\mathrm{N}=108)$

\begin{tabular}{|l|l|l|l|}
\hline Glycosaminoglycans' storage type & Number of patients & Frequency of thrombocytopenia & Platelet count, x10 $/ \mathrm{L}^{*}$ \\
\hline $\begin{array}{l}\text { MPS I, II, III (with heparan sulfate } \\
\text { storage) }\end{array}$ & 93 & $20 / 93(22 \%)^{\S}$ & $\begin{array}{l}121[95 ; 139] \\
0-147\end{array}$ \\
\hline $\begin{array}{l}\text { MPS IVA, VI (without heparan sulfate } \\
\text { storage) }\end{array}$ & 15 & $0 / 15(0 \%)^{\S}$ & - \\
\hline All patients & 108 & $20 / 108(19 \%)$ & $\begin{array}{l}121[95 ; 139] \\
0-147\end{array}$ \\
\hline
\end{tabular}

Footnote.*median [IQR], min-max platelet count, that is beyond the reference range; $\$ \mathrm{p}=0,068$ (Fisher's two-tailed exact test)

\section{Conclusion}

In conclusion, it is reasonable to state that thrombocytopenia is not a rare manifestation in patients with MPS. The autoimmune variant of HIT is one of the probable explanations of the disturbance. Thus the practical approach to patients with MPS, especially I, II, and III, must include regular complete blood count with platelet measurement, and immunomodulatory/immunosuppressive therapy should be considered in cases of thrombocytopenia, requiring treatment. Furthermore, additional analysis of laboratory HIT markers in MPSs is a perspective of searching new markers of clinical manifestations and severity of these lysosomal storage diseases.

\section{Abbreviation}

MPS(s): Mucopolysaccharidosis (es); GAGs: Glycosaminoglycans; HIT: heparin-induced thrombocytopenia; HLA: human leukocyte antigens; PF4: platelet factor 4; IQR: interquartile range

\section{Declaration}

Acknowledgment

None.

\section{Funding}

The authors received no financial support for their research, authorship, and/or publication of this article.

Availability of data and materials

Data will be available by emailing liliya-o@yandex.ru
Authors' contributions

Authors equally participated in the concept, design, writing, reviewing, editing, and approving of the manuscript in its final form. All authors have read and approved the final manuscript.

Ethics approval and consent to participate

We conducted the research following the Declaration of Helsinki. The Institutional Review Board of Federal State Autonomous Institution of the Ministry of Health of the Russian Federation "National Medical Research Center for Children's Health" jointly with the Academic Council of the Institution has approved the study (Reference number № 11 on 17 December 2013).

Consent for publication

Not applicable

Competing interest

The authors declare that they have no competing interest.

Open Access

This article is distributed under the terms of the Creative Commons Attribution 4.0 International License (http://creativecommons.org/licenses/by/4.0/), which permits unrestricted use, distribution, and reproduction in any medium, provided you give appropriate credit to the original author(s) and the source, provide a link to the Creative Commons license, 
and indicate if changes were made. The Creative Commons Public Domain Dedication

waiver (http://creativecommons.org/publicdomain/zero/1.0/) applies to the data made available in this article, unless otherwise stated.

\section{Author details}

${ }^{1}$ Consultative-diagnostic center, Federal State Autonomous Institution of the Ministry of Health of the Russian Federation "National Medical Research Center for Children's Health", Moscow, Russian Federation.

${ }^{2}$ Department of psychoneurology, Federal State Autonomous Institution of the Ministry of Health of the Russian Federation "National Medical Research Center for Children's Health", Moscow, Russian Federation.

Article Info

Received: 28 May 2021

Accepted: 01 August 2021

Published: 17 August 2021

\section{References}

1. Averianova NI, Rudavina TI, Domnina NA. Thrombocytopenia syndrome in a child with type II mucopolysacharidosis. Perm Medical Journal. 2014; 31(6):110-114 https://doi.org/10.17816/pmj316110-114.

2. Christian A, Watanabe $H$, Nakajima $T$, Inazu $T$. Idursulfase enzyme replacement therapy in an adult patient with severe Hunter syndrome having a novel mutation of iduronate-2-sulfatase gene. Clin Chim Acta. 2013; 423:66-68. https://doi.org/10.1016/j.cca.2013.04.022.

3. Cohen MA, Stuart GM. Delivery of anesthesia for children with Mucopolysaccharidosis Type III (Sanfilippo syndrome): a review of 86 anesthetics. Paediatr Anaesth. 2017; 27(4):363-369. https://doi.org/10.1111/pan.13075

4. Merdin A, Merdin FA, Karaca M, Güzelay N Mucopolysaccharidosis Type 3B in an Adult with Pancytopenia: A Rare Case Report. The Medical Bulletin of Haseki. 2014; 52(3):232-234. https://doi.org/10.4274/haseki.1614.

5. Muenzer J, Gucsavas-Calikoglu M, McCandless SE, Schuetz TJ, Kimura A. A phase I/II clinical trial of enzyme replacement therapy in mucopolysaccharidosis II (Hunter syndrome). Mol Genet Metab. 2007; 90(3):329-337. https://doi.org/10.1016/j.ymgme.2006.09.001.

6. Panigrahi I, Dhanorkar M, Didel S, Koganti RA. Hunter syndrome with persistent thrombocytopenia. BMJ Case Rep. 2019; 12(4):e226518. http://dx.doi.org/10.1136/bcr-2018-226518.

7. Ramaraj KP, NatarajanS, Ravikumar KG. Chronic idiopathic thrombocytopenic purpura in a child with Sanfilippo type A. Int J Contemp Pediatr 2015; https://dx.doi.org/10.18203/2349-3291.ijcp20150997.

8. Street AM. Hunter's syndrome with an endogenous anticoagulant. Am J Hematol. 1996; 53(4):277. https://doi.org/10.1002/(SICI)1096-8652(199612)53:4<277: AIDAJH15>3.0.CO;2-9.

9. Uz B, Demiroglu H, Ozcebe OI. Hunter syndrome and new onset idiopathic thrombocytopenic purpura in a young patient. Ann Hematol. 2012; 91(2):303-304. https://doi.org/10.1007/s00277011-1248-6.
10. Rajvanshi N, Kumar M. Immune thrombocytopenic purpura in a child with mucopolysaccharidoses. J Appl Hematol 2020; 11:135136. doi: 10.4103/joah.joah_26_20.

11. Baris HN, Cohen IJ, Mistry PK. Gaucher disease: the metabolic defect, pathophysiology, phenotypes and natural history. Pediatr Endocrinol Rev. 2014; 12 Suppl 1(0 1):72-81.

12. Coutinho MF, Lacerda L, Alves S. Glycosaminoglycan storage disorders: a review. Biochem Res Int. 2012; 2012:471325. https://doi.org/10.1155/2012/471325.

13. Lyon G, Kolodny EH, Pastores GM. Neurology of Hereditary Metabolic Disease of Children, Third Edition. McGraw-Hill, 2006. $-542 \mathrm{p}$

14. Eke S. Heparin-Induced Thrombocytopenia. [Internet]. New York: emedicine.medscape. c1994 -2021 [updated 2019 Dec 12, cited 2021 Jul 19]. Available from https://emedicine.medscape.com/article/1357846-overview\#a4

15. Tolar J, Orchard PJ, Key N, Blazar BR. Glycosaminoglycans as Anticoagulants in Mucopolysaccharidosis Type I (MPS I). Blood. 2007 ; $110(11): 2160.5$ https://doi.org/10.1182/blood.V110.11.2160.5.2160.5.

16. Tolar J, Orchard PJ, Key NS, Blazar BR. Circulating anticoagulant glycosaminoglycans in mucopolysaccharidosis type I. J Thromb Haemost. 2008; 6(5):893-895. doi: https://doi.org/10.1111/j.1538-7836.2008.02936.x.

17. Watson HA, Holley RJ, Langford-Smith KJ, Wilkinson FL, van Kuppevelt TH, Wynn RF et al. Heparan sulfate inhibits hematopoietic stem and progenitor cell migration and engraftment in mucopolysaccharidosis I. J Biol Chem. 2014; 289(52):3619436203. https://doi.org/10.1074/jbc.M114.599944.

18. Ahmad S, Haas S, Hoppensteadt DA, Lietz H, Reid U, Bender N et al. Differential effects of clivarin and heparin in patients undergoing hip and knee surgery for the generation of antiheparin-platelet factor 4 antibodies. Thromb Res. 2002; 108(1):4955. doi: 10.1016/s0049-3848(02)00397-3.

19. Bito S, Miyata S, Migita K, Nakamura M, Shinohara K, Sato T et al. Mechanical prophylaxis is a heparin-independent risk for antiplatelet factor 4/heparin antibody formation after orthopedic surgery. Blood. 2016; 127(8):1036-1043. https://doi.org/10.1182/blood-2015-06-651620.

20. Elshoury A, Khedr M, Abousayed MM, Mehdi S. Spontaneous heparin-induced thrombocytopenia presenting as bilateral adrenal hemorrhages and pulmonary embolism after total knee arthroplasty. Arthroplast Today. 2015; 1(3):69-71. https://doi.org/10.1016/j.artd.2015.07.003.

21. Warkentin TE. Knee replacement and HIT without heparin. Blood. 2016; 127(8):961-962. https://doi.org/10.1182/blood-201512-687665.

22. Warkentin TE, Cook RJ, Marder VJ, Greinacher A. AntiPF4/heparin antibody formation postorthopedic surgery thromboprophylaxis: the role of nondrug risk factors and evidence for a stoichiometry-based model of immunization. J Thromb Haemost. 2010; 8:504. doi: https://doi.org/10.1111/j.15387836.2009.03735.x.

23. Jurecka A, Żuberuber Z, Opoka-Winiarska V, Węgrzyn G, TylkiSzymańska A. Effect of rapid cessation of enzyme replacement therapy: a report of 5 cases and a review of the literature. Mol Genet Metab. 2012; 107(3):508-512. https://doi.org/10.1016/j.ymgme.2012.08.013.

24. Karnes JH, Shaffer CM, Cronin R, Bastarache L, Gaudieri S, James I et al. Influence of Human Leukocyte Antigen (HLA) Alleles and Killer Cell Immunoglobulin-Like Receptors (KIR) Types on Heparin-Induced Thrombocytopenia (HIT). Pharmacotherapy. 2017; 37(9):1164-1171. https://doi.org/10.1002/phar.1983. 